\title{
Uma discussão sobre os problemas da completude, da não-localidade e do realismo na teoria quântica
}

\author{
A discussion about the problems of completeness, non-locality, \\ and realism in quantum theory
}

\author{
RAFAELLE DA SILVA SOUZA \\ Instituto Federal de Educação, Ciência e Tecnologia da Bahia | IFBA \\ INDIANARA SILVA \\ Universidade Estadual de Feira de Santana | UEFS \\ ELDER SALES TEIXEIRA \\ Universidade Estadual de Feira de Santana | UEFS
}

RESUMO É apresentada uma narrativa histórica que representa um recorte dos arcabouços teóricos que ocupam lugar central no debate quântico e que deram lugar a várias interpretações da Mecânica Quântica (MQ). Discutimos, inicialmente, o argumento de Einstein, Podolsky e Rosen (1935) e os desenvolvimentos posteriores com foco no trabalho de John Bell (1964), que recolocaram as questões de fundamento teórico para o campo, também, experimental. De frente ao argumento que teve maior importância na busca de uma interpretação da $\mathrm{MQ}$, em conformidade com o período, destacamos a Interpretação de Copenhagen e sua coerência com a natureza com os fenômenos quânticos. Essa narrativa histórica é respaldada nos requisitos teórico-metodológicos da nova historiografia da história das ciências. Utilizamos textos originais como fontes primárias e várias outras secundárias que discorrem entorno do argumento de EPR. Uma possibilidade contribuição desse artigo é a utilização por estudantes/professores de física e entusiastas da teoria quântica, tendo em vista que, apesar da importância das discussões sobre a manutenção do determinismo, realismo e não-localidade, quase todos os livros de texto voltados a aprendizagem da MQ, com raras exceções, ignoram relevantes aspectos - em consequência, supre de algum modo lacunas quanto falta de materiais didáticos.

Palavras-chave Mecânica Quântica - Interpretação de Copenhagen - Narrativa Histórica

\begin{abstract}
A historical narrative is presented that represents a snapshot of the theoretical frameworks that occupy a central place in the quantum debate and that gave rise to several interpretations of Quantum Mechanics (QM). Initially, we discussed the argument of Einstein, Podolsky and Rosen (1935) and the later developments focusing on the work of John Bell (1964), which returned the questions of theoretical foundation to the field, also, experimental. Facing the argument that was most important in the search for an interpretation of $Q M$, in accordance with the period, we highlight the Copenhagen Interpretation and its coherence with nature with quantum phenomena. This historical narrative is supported by the theoretical and methodological requirements of the new historiography of the history of science. We use original texts as primary sources and several other secondary ones that run around the EPR argument. A possible contribution of this article is the use by students / professors of physics and enthusiasts of quantum theory, considering that, despite the importance of the discussions on the maintenance of determinism, realism and non-locality, almost all textbooks aimed at QM learning, with rare exceptions, ignores relevant aspects in consequence, it somehow fills gaps regarding the lack of teaching materials.
\end{abstract}

Keywords Quantum Mechanics - Copenhagen Interpretation - Historical Narrative 


\section{Introdução}

Historicamente, a Mecânica Quântica (M0), enquanto uma teoria cientifica formal, compreende dois momentos bem demarcados em sua constituição. 0 primeiro, marca o período entre 1900-1925 que se configura a partir de uma coleção de resultados experimentais obtidos na transição entre os séculos XIX e XX, são exemplos: duplicação de linhas espectrais em campo magnético; ionização por raio X; medição de energias de elétrons secundários; emissão e absorção de luz em quantidades discretas de energia; experimento da dupla fenda para luz fraquíssima; calores específicos dos sólidos; espalhamento de raios alfa pelo núcleo; efeito fotoelétrico; duplicação de linhas em campo elétrico; perda de energia de elétrons movendo-se em vapor de mercúrio; efeito Compton e etc. Trata-se de resultados que a Mecânica Clássica, nos moldes em que foi configurada entre os séculos XVIII e XIX, não podia explicar.

Hoje, este período é conhecido como a velha teoria quântica - ou ainda, antiga teoria quântica. A partir de 1927, o formalismo da teoria quântica foi estabelecido formalmente, para descrever o comportamento das entidades subatômicas, e se mantém até hoje em ascensão - o que configura a M0 propriamente dita, ou ainda, M0 moderna. Passados os anos efervescentes que serviram de pano de fundo para o desenvolvimento (1900-1922) e formulação (1923-1928), veio o segundo momento de demarcação, período de desdobramentos, iniciado na década de 30. Neste a teoria torna-se de fato um paradigma, se legitimando pela capacidade de gerar explicações para uma gama de fenômenos cada vez mais amplos como na física nuclear, na física do estado sólido, no vigoroso desenvolvimento da teoria quântica de campos e nas inovações tecnológicas etc.

Meados da década de 30, pode-se dizer que especialistas relegaram para segundo plano as questões epistemológicas, ou assuntos filosóficos correlatos. As discussões sobre os fundamentos e interpretações ficaram restritas a pequenos grupos e a filósofos. Um trabalho que ganha destaque é o de Bell, em 1964느, que recolocou as questões fundamentais e filosóficas num novo patamar, onde podia ser investigada também experimentalmente ${ }^{2}$. Ao longo dos anos, 0 debate sobre interpretações fica a cargo de Copenhague, sendo ortodoxia, e o ressurgimento da interpretação de De Broglie-Bohm e muitos mundos, além disso, várias outras interpretações surgiram nos anos 1980 como, por exemplo, a interpretação das histórias consistentes ${ }^{3}$. Pessoa Jr. ${ }^{4}$, apresenta um estudo geral que envolve quarenta interpretações da teoria quântica.

Agora, com mais de 100 anos de crescimento, ao nos interessarmos pela história da teoria quântica, seja por interesse pessoal ou para preparar material didático, nos deparamos com a questão: onde buscar fontes confiáveis. Uma possibilidade é buscar boas referências históricas como o livro de Mehra e Rechenberg ${ }^{5}$, intitulado The Historical Development of Quantum Theory; ou clássicos que tratam da história e dos problemas conceituais da teoria quântica ${ }^{6}$, ou, ainda, o livro de Olival Freire Jr. The Quantum Dissidents, publicado em 2015.

Nesse caso, uma impressão que pode surgir é que vem uma avalanche de nomes, filiações, teorias e experimentos. Infelizmente, e talvez porque se trata de uma complexa teoria e/ou por não se ter intimidade com o assunto, o contato histórico via tais livros pode parecer confuso ou muito complexo.

Outra possibilidade é buscar referências bibliográficas clássicas como: Eisberg e Resnick; Tipler e Llewellyn; Cohen-Tannoudji, Diu e Laloë ${ }^{8}$; Caruso e Ogurịi; Griffiths ${ }^{10}$; entre outros. Estes livros, tradicionalmente presentes em cursos de graduação, estão longe de oferecer o que vem sendo argumentado para uma melhor formação histórico-conceitual da teoria quântica. São organizados tematicamente, em ordem cronológica, sem considerar que o conhecimento científico não é construído de tal forma ${ }^{11}$.

Uma terceira opção são as narrativas históricas que têm sua importância por demonstrar os encaminhamentos e a exploração de fontes históricas de um determinado episódio histórico, capazes de mobilizar a aprendizagem histórica e auxiliar na formação de professores e estudantes. São exemplos de narrativas, que seguem tendências historiográficas baseadas na história da Espectroscopia Atômica e de vários tópicos da Física Estatística, como radiação do corpo negro ou da estatística de gases: Hendry ${ }^{12}$, Beller ${ }^{13}$ e Darrigo ${ }^{14}$. 
Essa tendência é particularmente significativa, inclusive para as disciplinas científicas, conforme argumentam Millar e Osborne ${ }^{15}$ e Norris e colaboradores ${ }^{16}$. Para estes autores, as narrativas passam a ter um importante papel: favorecer a apresentação de conteúdos científicos e de ideias num contexto social, histórico e cultural mais amplo $0^{17}$. As narrativas delimitam um estudo histórico com mais clareza e descreve ações, experiências feitas por um certo número de personagens, bem como sua contribuição na construção do conhecimento ${ }^{18}$.

Diante das possibilidades, por reconhecer a amplitude dos conhecimentos sobre a teoria quântica, este trabalho faz uma opção pela da utilização de narrativas voltada para o ensino de ciências. Empreendemos esforços na escrita de uma narrativa histórica que envolve um recorte temático acerca de algumas discussões sobre o problema da completude, o conceito da não-localidade e o critério epistemológico do realismo. A expectativa é elucidar questionamentos e influências em relação aos desdobramentos na teoria.

0 problema da completude, apresentado inicialmente por von Neumann ${ }^{19}$ e depois no artigo de Einstein, Podolsky e Rosen, em 1935, foi discutido por uma minoria interessada nos fundamentos da teoria quântica, mas foram amplamente ignorados pela grande massa de professores do nível superior deixando, quase sempre, fora do currículo. Inclusive, os livros usados nos cursos, até recentemente, apresentam apenas a interpretação de Copenhague, sem jamais abordar a discussão profunda e riquíssima que os autores destes artigos e outros da época levantaram²0. No entanto, consideramos que divulgar discussões fundamentais da teoria são importantes e podem contribuir para um enriquecimento do ensino de física.

Por sua vez, sabemos que não é elementar escrever uma narrativa histórica, que abarca uma infinidade de perspectivas e requer a definição de recortes bem delimitados para o enfoque, tanto do ponto de vista temporal, quanto temático, além da necessidade do cuidado com possíveis equívocos historiográficos que ocasionam problemas epistemológicos $\underline{21}$. É preciso atender aos requisitos historiográficos bem como às recomendações para a transposição didática $\underline{22}$.

Assim, consideramos os requisitos da nova historiografia das ciências: de qualificar o ensino através da contextualização que possa contribuir com maior precisão para os saberes científicos e tecnológicos da atualidade ${ }^{23}$. Tal escolha foi motivada pelos propósitos educacionais e os objetivos estabelecidos de pesquisa para uma tese de doutorado, para a qual tal narrativa foi inicialmente construída. A versão aqui apresentada configura material de leitura que fundamenta uma proposta didática para abordar tópicos da M0 na licenciatura em física. No entanto, sua utilização pode ser ampliada a todo leitor que tenha interesse na temática por ser composta de aspectos históricos sobre a teoria quântica, bem como seus desdobramentos na compreensão de certos aspectos da teoria que podem ser muito fecundos como estratégias de ensino acerca de conceitos que carecem de trivialidades.

\section{Referencial teórico-metodológico}

Uma narrativa histórica que explore elementos histórico-filosóficos constitui forte aliado ao ensino de ciências. Concordamos com autores como Norris e colaboradores ${ }^{24}$, Klassen ${ }^{25}$ e Metz e colaboradore ${ }^{26}$ que apontam nas narrativas um auxílio na geração de interesse em aprender, por parte do estudante, pois os incentivam a gerar questões sobre o conteúdo da mesma. Desse modo, a narrativa foi construída sendo o principal público-alvo estudantes de licenciatura em física.

Nossa pretensão é contemplar uma abordagem alternativa sugerida por Martins, baseada na ideia de temas no lugar de princípios através do eixo histórico-epistemológico. São agrupados temas relativos ao papel do indivíduo, da comunidade científica e as influências históricas e sociais; com temas relativos à origem do conhecimento (experiência $\mathrm{x}$ razão; papel da observação, da experiência, da lógica e do pensamento teórico; influência da teoria sobre o experimento), aos processos da ciência (coleta, análise e avaliação de dados; inferência, correlação e causalidade; modelagem em ciência; papel da imaginação e criatividade; natureza da explicação) e ao conteúdo da MQ²․ 
Concordamos com Allchin e colaboradores, ${ }^{28}$ que elencam alguns dos elementos essenciais que devem estar presentes em uma narrativa histórica. Em nosso caso, damos destaque para as perspectivas históricas que mostrem a incerteza da ciência, ou seja, que a ciência está em construção; e para questões que problematizem a natureza da ciência e promovam a investigação da natureza da ciência. A abordagem historiográfica adotada neste trabalho privilegia uma discussão centrada principalmente nos conceitos físicos e, por conta disso, orienta-se, por meio de um processo de análise crítica para discutir, interagir e questionar sobre a história da ciência apresentada.

Dessa forma, espera-se auxiliar no estudo da teoria quântica de forma ampla ao passo que tratamos do debate a respeito do realismo, da não-localidade e da completude da teoria quântica. Sabemos que a teoria suscitou diversos outros debates sobre questões de fundamentos, e consideramos o argumento de EPR um marco importante nos esclarecimentos das questões envolvidas nesse debate.

Este texto apresenta, inicialmente um contexto geral da teoria situando o período e os conhecimentos que se tinham na época. Em seguida, analisamos as fontes primárias dialogando com os trabalhos já existentes a respeito da temática escolhida, caminhando para a construção da referida narrativa. É apresentada uma breve introdução às interpretações da teoria quântica, feita de uma perspectiva histórica, sendo explorados alguns pontos discordantes, avanços teóricos e conceituais, chegando à validação experimental que conhecemos hoje. Em alguns casos, o texto traz trechos de fontes primárias, com descrições de experimentos ou com explicações dadas por cientistas do passado para determinados fenômenos físicos.

Parte da bibliografia trata-se de fontes primárias a serem analisadas. Outra parte é referente a trabalhos já existentes a respeito da interpretação dessas fontes e, portanto, estabelecemos um diálogo inicial com o artigo de Einstein, Podolsy e Rosen,, 29 terminando por centrar nas desigualdades de Bell sinalizando certos aspectos dos estudos de Schrödinger ${ }^{30}$, Bell ${ }^{31}$ e Aspect e colaboradores $\underline{32}$ - todas fontes primárias. Desse modo, esperamos trazer novas contribuiçõos a respeito dos debates em torno da questão de completude, da não-localidade e do realismo na teoria quântica.

\section{Do quantum ao argumento de EPR}

Em meados da década de 1920, a compreensão do nível subatômico incorpora a conceituação de quantum nos fundamentos da física. Estava claro que elétrons, átomos, moléculas, há muito considerados como partículas também mostram propriedades ondulatórias. Firma-se nesse momento o conhecimento que estabelecia a antiga teoria quântica, abrindo espaço para o surgimento de novas conceituações - como, o abandono do determinismo inerente à física clássica e aceitação de uma descrição essencialmente probabilista. Em 1923, tinha-se uma teoria quântica que era insatisfatória e vários resultados experimentais requerendo explicação ${ }^{33}$.

A princípio, a teoria apareceu em diferentes formalismos matemáticos e com propostas independentes. Em resumo, Heisenberg forneceu a base para M0 enfatizando o aspecto da partícula dos objetos quânticos e Schrödinger enfatizou 0 aspecto da onda. Para Schrödinger, de fato, essas duas formas da M0 são equivalentes. Isto é, maneiras diferentes de expressar as mesmas relações. A equação de Schrödinger tornara-se predominante, embora os símbolos usados sejam, agora, interpretados de forma diferente do uso original ${ }^{34}$.

Entre 1925 e 1926, intervalo de tempo de dois anos, Heisenberg, Max Born e Pascual Jordan formularam a M0 para uma interpretação corpuscular (enquanto positivista), representando-a em forma de matrizes. Paul Dirac, através de uma outra representação, formulou, de forma independente, a nova teoria. Schrödinger, também de forma independente, associando à física ondulatória clássica, obteve a equação que leva seu nome e que constitui um dos postulados da $\mathrm{M0}$, fornecendo a função do estado $\psi$ em função do tempo e admitindo uma interpretação ondulatória. Enquanto, Bohr, em 1927, defendia uma interpretação claramente dualista que punha os aspectos de onda e partícula como complementares - conhecida com interpretação da complementaridade. $\frac{35}{5}$ 
Outros físicos de destaque do século XX, entre os quais Planck, Einstein, De Broglie, Pauli e Feynman, têm o nome, de algum modo, associado à MQ ${ }^{36}$ Na sequência, os fenômenos microscópicos passam a ser vistos de modo dualista, o que ficou amplamente conhecido como "interpretação ortodoxa" da MQ, sustentada por Bohr e por um conjunto de cientistas. $\stackrel{37}{ }$

Discussões memoráveis são observadas, tendo um marco no $5^{\circ}$ Congresso Solvay, em 1927, sobre os aspectos quânticos da matéria. Uma discussão que merece destaque, que se prolonga até 1930, envolveu as tentativas de Einstein para escapar ao princípio de incerteza através de uma violação para energia e tempo. No entanto, Bohr, vence a discussão apontando os erros de Einstein e ganhando mais adeptos a interpretação ortodoxa da $\mathrm{MQ}^{33}$.

Em 1932, John von Neumann, representa estados quânticos em um espaço vetorial complexo (espaço de Hilbert), discute o problema da medição para um sistema quântico e conclui que o resultado de uma medição é indeterminado porque 0 estado do observador antes da medição não é conhecido de maneira exata. Von Neumann tenta provar a impossibilidade da teoria de variáveis ocultas, mas a prova acaba por ter algumas lacunas que são, posteriormente, discutidas por Wigner ${ }^{\underline{39}}$ e Bell|ㅂ. 0 que parece uma limitação absurda da natureza, devido a imprevisibilidade dos resultados, da origem aos processos de abandono do determinismo clássico.

Sobre a medição da posição e momentum de duas partículas não-interagentes, mas correlacionadas, Einstein juntamente com o ucraniano Boris Podolsky e o americano Nathan Rosen, colegas da Universidade de Princeton, apresentam um artigo que deu origem ao famoso argumento de EPR (ulteriormente chamado EPR, representando as iniciais dos autores) ${ }^{41}$. Este trabalho provocou primeiro esclarecimentos fundamentais da $\mathrm{MO}$ envolvendo estados ditos correlacionados (i.e., que interagiram no passado) ou "emaranhados" e, depois, motivou atividades experimentais que exibiram a existência de tais estados. A função de onda de uma única partícula não poderá ser escrita como um produto de funções de onda de partículas individuais e, portanto, o emaranhamento é entendido como uma característica genérica de duas funções de onda de partícula.

Einstein, Podolsky e Rosen levantaram um dos problemas mais fundamentais da teoria quântica: seria a MQ uma teoria completa ou se, pelo contrário, contém variáveis escondidas. Para os autores, a posição e o momentum de uma das partículas poderiam ser considerados elementos simultâneos de realidade, já que os valores de cada um desses observáveis poderiam ser determinados, sem perturbar de qualquer maneira o sistema, simplesmente medindo a posição ou o momentum da outra partícula.

Nessas configurações, admite-se a "incompletude" da M0 e questiona-se a interpretação de Copenhagen. De acordo com a MQ, duas observáveis cujos operadores não comutam não podem ter realidade simultânea. Conceitualmente simples, mas fugindo à vivência clássica de uma teoria de partículas, um dos aspectos relacionados ao EPR é a não-localidade, o que os autores do citado trabalho descartaram como hipótese de trabalho válida. Esses argumentos configurou uma longa controvérsia envolvendo cientistas e filósofos tendo atingido seu ápice em 1982, quando se verifica experimentalmente a questão da não-localidade.

No mesmo ano, Bohr프 refina a interpretação da complementaridade, dando importância ainda maior para 0 aparelho experimental, cujo arranjo passou a determinar se o "fenômeno" observado exibe características de partícula ou de onda. E, também, Schrödinge ${ }^{43}$ que apresentou seu experimento mental do paradoxo do gato e adota uma postura mais "realista" para usar o problema da medição como um argumento para a incompletude da MO.

Anos mais tarde, David Bohm, em 1952, reinterpreta a teoria quântica reproduzindo um amplo quadro conceitual que, com o auxílio de parâmetros adicionais (variáveis ocultas), permite nova descrição dos sistemas quânticos no qual, qualquer partícula teria posição e momentum simultâneo bem definidos, e estas variáveis evoluiriam de forma contínua e determinística. Assim, o problema da completude é resolvido através de uma modificação do formalismo quântico semelhante ao que ocorre na teoria da onda piloto de De Broglie ${ }^{44}$.

Outra solução radical ao problema geral da medição seria a abordagem dos "muitos mundos" de Everett, em 1957, que postulou que o universo como um todo deveria ser descrito por uma única função de onda que evoluiria de modo 
determinístico de acordo com a equação de Schrödinger. Embora fosse perceptível diferentes interpretações para os fenômenos quânticos, a interpretação de Copenhagen foi estabelecida com segurança e poucos estavam interessados em reconsiderar seus fundamentos ${ }^{45}$. Esse debate, no entanto, sempre continuou vivo, tendo surgido posteriormente novos elementos para a discussão.

Por exemplo, pouco tempo depois, Bell estabelece as desigualdades e mostra que teorias desse tipo são necessariamente não-locais ${ }^{46}$. Em 1964, com uma atitude realista defendendo a teoria de variáveis ocultas, estabeleceu as desigualdades de Bell em coeficientes de correlação de spin a partir da localidade de Einstein para correlações EPR, compartilhando os mesmos pressupostos de 1935. A década de 1960, marca um novo capítulo na M0, mais um passo, e desde então continuou a evoluir. Com passar do tempo, as desigualdades são testadas experimentalmente com precisão e sofisticação tendo uma profunda influência sobre o campo de fundamentos da M0.

As desigualdades de Bell fornecem um critério simples para testar qualquer teoria local de fenômenos naturais, formulada sob o marco do realismo e evidenciam que a M0 não é uma delas. Ao discutir o teorema de Bell, dar-se destaque ao dilema (ou "trilema") existente: ou abandona-se o realismo, ou abandona-se a localidade (ou os dois).

0 teorema de Bell foi apenas o preâmbulo de muitas atividades promissoras que discutiam o realismo nos últimos 50 anos. Por exemplo, em 1969, os físicos John Clauser e Abner Shimony perceberam que os resultados experimentais disponíveis permitiam verificar a dupla escolha implícita no teorema de Bell: seja a teoria quântica ou realismo local ${ }^{47}$. Desde então, uma série de experiências foram realizadas levando a confirmação dessa estranha propriedade quântica: a não-localidade. Nos anos 80, os físicos conseguiram manipular fótons, elétrons, nêutrons e átomos um a um, tornando assim todos esses experimentos idealizados reais, promovendo desenvolvimentos teóricos combinados com avanços experimentais.

Cabe acrescentar que, testes experimentais das desigualdades de Bell, apresentam resultados favoráveis à M0 e ganharam destaque na década de 70 e 80 . Esses experimentos são realizados primeiro por Freedman e Clauser ${ }^{48}$, depois por muitos outros, e sustentou consistentemente as previsões da $\mathrm{MQ}$, tornando mais difícil de sustentar qualquer tipo de teoria da variável escondida local. Fecham as lacunas os experimentos de Aspect et al. ${ }^{49}$ - posteriormente, verificado por outros. Sobre a cultura material dos primeiros testes experimentais do teorema de Bell, sugerimos a leitura do trabalho de Bispo e David $\underline{50}$ que analisa algumas técnicas e instrumentos.

Em continuidade, características da M0 como o emaranhamento consideradas estranhezas perturbadoras, são agora, sistematicamente, exploradas. Em termos de aplicações, os problemas conceituais levantados fizeram com que se desenvolvessem métodos de observação dos objetos quânticos que esclareceram muito a respeito do seu comportamento. Viu-se um rápido aumento no interesse na computação quântica e na comunicação quântica, em que as propriedades quânticas de objetos microscópicos podem ser usadas como uma parte essencial dos processos de informação. É entendido, que o argumento da incompletude só funciona com a imposição da localidade. Com essa percepção, 0 emaranhamento quântico não pode ser reduzido à dualidade onda-partícula: é, de fato, um novo mistério e é tido hoje como o segundo aspecto fundamental da teoria quântica ${ }^{51}$.

Todo esse processo, sumariamente descrito, fornece importantes aspectos para o estudo histórico-conceitual da M0. Obviamente, não é possível descrever com rigor toda a história da M0 em poucas linhas, essa história vai além do escopo aqui apresentado. Existem inúmeras fontes sobre o assunto (algumas já supracitadas). Nossa intenção é apresentar uma discussão em torno do abandono do determinismo, passando pelo argumento de EPR e alguns aspectos cruciais que deste surgiu.

Ressaltamos, portanto, a importância do argumento de EPR: estabelecido em 1935, fomentou conflito na M0 com questões em relação à completude da teoria. A partir da Teoria de Variáveis Ocultas Locais (TVOL), que surgiu em 1965, e as desigualdades de Bell - peça principal na resolução da controvérsia - guia-se à realização dos experimentos e desdobramentos teóricos. Considerando esse contexto, nas seções seguintes iremos nos aprofundar nos argumentos de Einstein, Podolsky e Rosen. 


\section{O artigo de EPR e a (in)completude da MQ}

0 artigo intitulado “Can Quantum Mechanical Description of Physical Reality Be Considered Complete?", publicado na revista Physical Review, atraiu atenção da comunidade científica daquela época, justamente pelo fato de colocar em xeque a M0. Através de um experimento de pensamento (Gedankenexperiment), Einstein, Podolsky e Rosen supostamente mostravam que a M0 era uma teoria incompleta, apesar de não fazerem referência a uma possível maneira de completá-la.

Há uma insistência na localidade e no realismo. 0 argumento apresentado discutia a descrição quântica da realidade física fornecida pelas funções de onda alicerçadas na condição de completude - para cada elemento da realidade física deve existir um elemento que o represente na teoria - e se consolidava em duas perguntas que os autores julgaram importantes para verificar o sucesso de uma teoria. A primeira "a teoria é correta" e a segunda "se a descrição fornecida pela teoria é completa".

Para verificar se uma teoria é correta suas conclusões devem estar concordando com as experiências humanas. No campo da física essa verificação é feita através da experimentação, ou seja, da mensuração. Contudo, esta primeira pergunta não foi levada em consideração pelos autores para a construção de seus argumentos, na época a M0 era considerada correta em suas previsões. Todo argumento foi desenvolvido considerando apenas a segunda pergunta, assim, para que uma teoria seja considerada completa deve satisfazer a condição de completude.

A pré-história das desigualdades de Bell encontra-se na crítica feita por EPR à interpretação de Copenhagen, considerada a crítica "madura" de Einstein ${ }^{52}$. É que anteriormente ele buscava, através de Gedankexperimenten, limitar a validade das relações de Heisenberg. Do início dos anos de 1930 em diante Einstein não mais busca este objetivo, mas passa a considerar a teoria quântica como logicamente consistente, de forte respaldo empírico, mas incompleta. Essa percepção é estabelecida mediante duas condições fundamentais: condição de completude e critério de realidade física ${ }^{\frac{53}{3}}$. Voltaremos a examinar essas condições nas seções seguintes.

Contudo, essa argumentação não aparece tão explícita no artigo de 1935. Os autores propõem um experimento que revelaria existirem propriedades físicas dos microobjetos que não estariam expressas no formalismo da teoria, daí sua incompletude. 0 experimento proposto por EPR consiste em:

Para este efeito, suponhamos que temos dois sistemas, I e II, que permitem a interação do tempo $t=0$ para $t=T$, após o que supomos que não há mais nenhuma interação entre as duas partes. Supomos também que os estados dos dois sistemas antes de $t=0$ já eram conhecidos. Podemos então calcular, com a ajuda da equação de Schrödinger, o estado combinado do sistema I + II em qualquer momento posterior, em especial, para qualquer $t>T$. Vamos designar a função de onda correspondente por $\psi$. Não podemos, no entanto, calcular o estado em que qualquer um dos dois sistemas é deixado após a interação. Isso, segundo a Mecânica Quântica, só pode ser feito com a ajuda de outras medições, por um processo conhecido como a redução do pacote de ondas. $\underline{54}$

Entendemos que um sistema composto de dois objetos quânticos interage num dado instante e depois separam-se espacialmente. É realizada então uma medição de um determinado observável físico em uma das partículas. Com essa informação mais a função de estado do sistema das duas partículas (fornecida pela teoria quântica) e mais algumas informações derivadas da interação que teria ocorrido entre as duas partículas, podemos prever o valor do mesmo observável para a outra partícula, que não foi objeto de medição. Logo, o observável da segunda partícula pode ser conhecido sem ser objeto de medição. Ao representar a função de estado podemos optar por medir outro observável físico na primeira partícula e, pelo mesmo processo, poderemos conhecer o valor deste outro observável para a segunda partícula sem realizar qualquer medição sobre esta. A opção por medir um ou outro observável não altera a função de onda. Mas para facilitar o cálculo das probabilidades, é conveniente mudar a base de representação. 
É possível então afirmar a existência de distintos observáveis da segunda partícula sem realizar qualquer medição sobre ela. Isto é, usando o princípio da localidade, podemos afirmar que para a segunda partícula os observáveis estavam predeterminados, tinham valores precisos, independentes de qualquer medição.

Vemos que, como consequência de duas medições diferentes efetuadas sobre o primeiro sistema, o segundo sistema pode ser deixado em estados com duas funções de onda diferentes. Por outro lado, uma vez que no momento da medição os dois sistemas não interagem, nenhuma mudança real pode ocorrer no segundo sistema em consequência de qualquer coisa que possa ser feito ao primeiro sistema. Esta é, naturalmente, apenas uma declaração do que se entende pela ausência de uma interação entre os dois sistemas. Assim,

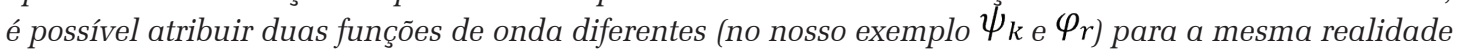
(o segundo sistema após a interação com o primeiro). $\frac{55}{5}$

No experimento proposto, considera-se os dois observáveis físicos canonicamente conjugados como sendo a posição e o momentum das duas partículas no formalismo da teoria quântica. Como neste formalismo, estes observáveis não podem ter realidades simultâneas, chegamos a uma situação em que o sistema físico teria propriedades (os dois observáveis canonicamente conjugados da segunda partícula) não admitidas pelo formalismo da teoria.

É estabelecido um formalismo incompleto, porque não incorporava, em seu formalismo, todas as propriedades existentes na realidade física. Vale destacar que uma teoria capaz de expressar no seu formalismo variáveis canonicamente conjugadas, como posição e momentum, teria como consequência a restauração do determinismo próprio da física clássica, o que era também uma aspiração de Einstein. Vamos discutir separadamente cada uma das afirmações feitas por EPR.

\section{Descrição da Realidade (primeira afirmação)}

Em termos lógicos e utilizando-se do realismo, o formalismo da MQ, que inclui o "postulado da projeção" e descreve a redução de estado, podemos prever algo com certeza, então existe um "elemento de realidade" correspondente. 0 conceito de realidade, condição "suficiente" de realidade, dada pelos autores no artigo é que: "se, sem de modo algum perturbar o sistema, pudermos prever com certeza (ou seja, com probabilidade igual a um) o valor de uma quantidade física, então existe um elemento de realidade física correspondente a esta quantidade física" $\underline{.6}$

Este conceito não esgota as maneiras de se reconhecer uma realidade física, mas fornece uma dessas maneiras e, em certas condições específicas, está em concordância tanto com as ideias clássicas de realidade como com as ideias quânticas. De forma mais geral, demonstra-se que na M0 o conhecimento preciso de um observável implica na indeterminação do outro (para o caso de observáveis canonicamente conjugados, como posição e momentum, energia e tempo, etc.), mais ainda, qualquer tentativa de determinar experimentalmente um observável altera o estado da outra.

A primeira afirmação feita é que: "(1) a descrição da realidade dada pela função de onda na mecânica quântica não é completa, ou (2) estas duas quantidades não podem ter realidades simultâneas"..$\underline{57} 0$ que se entende é que, para a M0, quando duas partículas são afastadas por certa distância e se efetua uma medição do momentum de uma dessas partículas, isso modifica o resultado da medição do momentum da outra partícula - essa ideia é hoje conhecida como não-localidade. A ideia de localidade é justamente o contrário, efetuando-se uma medição do momentum em uma partícula não modifica o resultado da medição da outra. Com isso, argumentando apenas pela possibilidade de se realizar medição, EPR admitem a incompletude da teoria quântica.

\section{A contradição (segunda afirmação)}

Quando se considera que a função de onda fornece uma descrição completa da realidade, dentro do contexto das ideias discutidas no artigo, surge uma contradição (mostrado através do experimento citado na seção anterior) $\frac{58}{\text {. }}$. 
Ao considerar o processo descrito, por exemplo, se um sistema qualquer antes da medição é descrito pela superposição de dois autoestados $\Psi_{1}$ e $\Psi_{2} \Psi=\Psi_{1} C_{1}+\Psi_{2} C_{2}$ onde $C_{1}$ e $C_{\text {, são as amplitudes complexas, sendo }{ }^{59}}$ $\Sigma\left|C_{i}\right|^{2}=1$, após a medição o sistemá se reduz a um dos dois estados $\Psi_{1}$ ou $\Psi_{2}$, fala-se de colapso da função de onda ${ }^{60}$. Sobre a noção de colapso da função de onda, apenas algumas interpretações da teoria quântica consideram como consequência do formalismo da MO.

Em consequência de uma medição executada sobre I, o sistema II pode ter duas funções de ondas diferentes. Por outro lado, no ato da medição os sistemas não estão mais interagindo, "não pode ocorrer nenhuma mudança real em II, em decorrência de qualquer alteração que se faça em I". 61 Logo, é possível atribuir duas funções de onda diferentes à mesma realidade física.

Retomemos a primeira afirmação, partindo da suposição de que a função de onda fornece uma descrição completa da realidade, os autores mostram no referido artigo que podem existir realidades físicas simultâneas para esses dois observáveis, constituindo assim um paradoxo. Então, os autores afirmam: "somos assim forçados a concluir que a descrição quântica da realidade física através das funções de onda não está completa". $\underline{62}$

Desta forma, 0 artigo é finalizado sem discutir sobre a existência ou não de uma descrição completa da teoria quântica deixando esta questão em aberto. 0 realismo de Einstein 0 influenciou em sua crítica à teoria quântica ${ }^{63}$. Segundo Chibeni ${ }^{64}$, há no trabalho um apelo para a interpretação de ortodoxa ao afirmar que, para pares de quantidades físicas incompatíveis, que: "o conhecimento preciso de uma impossibilita o conhecimento preciso da outra e, que a tentativa de determinar uma delas alterará o estado da outra" $\underline{\underline{65}}$ Nesse caso, ao analisar a condição de completude e o caráter não determinístico da teoria, percebe-se claramente que ambos não se suportam na M0, e significa a violação do determinismo, pois trata o colapso do sistema para um estado definido como sendo aleatório e dependente de uma medição.

Muita discussão foi gerada pelo argumento de EPR, inclusive Bohr ${ }^{66}$ com bastante cautela responde a este artigo com outro de mesmo título e aprimora a interpretação da complementaridade. Na perspectiva de Bohr, 0 argumento de EPR não continha nenhum desafio de ordem prática para a aplicação da teoria quântica a problemas físicos reais ${ }^{67}$. Nos anos 60 o problema retornaria sob outra roupagem, através da desigualdade de Bell.

\section{A participação de Schrödinger em 1935}

No mesmo ano, Schrödinger $\underline{68}$ apresentou de modo dramático as consequências do princípio da superposição voltado a discussão do problema da medição. Através do experimento mental do gato - Gedankenexperiment - , embora não envolva a hipótese da localidade (e nem supõe a não-localidade), contribuiu de forma indireta com o abandono do determinismo pelo fato de discutir a interpretação de Copenhague e o comportamento da superposição de estados até ser observado ou interagir com o mundo externo. Schrödinger mostrou que poderíamos ter situações no mínimo estranhas caso o mundo macroscópico fosse totalmente descrito pela M0.

Um gato é colocado numa câmara de aço, juntamente com o seguinte dispositivo diabólico (protegido contra a interferência direta do gato): um contador Geiger com uma pequena quantidade de substância radioativa, tão pequena, que talvez no curso de uma hora um dos átomos decai, mas também, com igual probabilidade, talvez nenhum; se isso acontecer, o tubo contador descarrega e através de um relé libera um martelo que quebra um pequeno frasco de ácido cianídrico. Se não tocarmos no sistema durante uma hora, diríamos que o gato ainda vive se, enquanto isso, nenhum átomo tiver decaído. O primeiro decaimento atômico teria envenenado o gato. A $\Psi$-função de todo o sistema expressaria isso tendo nele o gato vivo e morto (perdoem a expressão) misturadas ou sobrepostas em partes iguais. ${ }^{69}$ 
Seguindo Schrödinger, imaginemos um gato dentro de uma caixa fechada. 0 gato pode estar vivo (|vivo $\rangle$ ) ou morto $(\mid$ morto $\rangle)$. Dentro da caixa, há um átomo instável $\left(\left|a_{\text {tomo }}\right\rangle\right)$, que pode ter decaído para um outro átomo mais estável $\left(\left|a ́ t o m o_{2}\right\rangle\right.$ ). Suponhamos que a energia liberada nessa transição é suficiente para ativar um mecanismo que libera um gás tóxico, matando o gato. A MQ prevê a probabilidade de o isótopo instável decair, sem nos dizer quando isso ocorre (indeterminismo). Assim, aceitando a validade do princípio da superposição, o estado do sistema composto pelo gato e pelo átomo é

$$
\left.\left.\left.\left.|\Psi\rangle=a \mid \text { átomo } o_{1}\right\rangle \mid \text { vivo }\right\rangle+b \mid \text { átomo }{ }_{2}\right\rangle \mid \text { morto }\right\rangle
$$

Aqui $|a|^{2}$ e $|b|^{2}$ nos dão as probabilidades de 0 átomo não decair e decair, respectivamente.

0 estado acima é uma superposição de dois estados macroscópicos distintos do gato: superposição dos estados de "vivo" e "morto" (simultaneamente). Este resultado, absurdo classicamente, enfatiza a questão sobre o adequado limite entre sistemas clássicos e quânticos. Schrödinger, também explicita o problema da medição que surge quando se investiga mais detalhadamente essa forma de tratar a teoria.

Para enfatizar a conexão íntima entre os dois subsistemas (gato mais átomo), Schrödinger introduziu na física o termo Verschränkung, o qual, por sua vez, foi traduzido do alemão para o inglês como Entanglement e, finalmente, para o português como emaranhamento. 0 emaranhamento surge ao analisar o argumento de EPR (que envolve um sistema entrelaçado de duas partículas, 1 e 2), ao se questionar: como medidas em uma partícula podem influenciar a outra se elas estão suficientemente distantes a ponto de não haver interação (da amostra com o medidor); ou, como gera correlações não locais que não podem ser explicadas classicamente. A partir daí surgiram intensos debates filosóficos sobre 0 que exatamente seria o emaranhamento, pois sua compreensão vai depender da interpretação filosófica da $\mathrm{MO}$ a que se estiver assumindo.

Ainda sobre à situação de estado do gato, a princípio não se parece aceitável acreditar em um objeto macroscópico em uma superposição de estados, inclusive, ainda não há relatos disso. A passagem do mundo microscópico para 0 macroscópico não é nada trivial e ficou conhecido como um processo de descoerência quântica.

Uma justificativa imediata dentro do fenômeno de descoerência é que a observação do sistema quântico ocorre no momento em que 0 sinal é ativado no detector, o observador lê a medida transformando o estado em um autoestado do dispositivo, existindo assim um mecanismo que separa o estado clássico do gato (e de todos os componentes macroscópicos) e o estado quântico do sistema que evolui em superposição. Surgem então outras questões: qual o limite que diferencia um objeto capaz de atuar como um observador quântico de um objeto quântico por si (restrito à condição de observado)? 0 que precisamente constitui a medida? Estas perguntas são muito difíceis de serem respondidas com clareza de forma a não gerarem outras indefinições de compreensão.

Outra questão, é a possibilidade da observação quântica depender da presença de um observador consciente, colocando em evidencia aspectos do caráter subjetivo da compreensão da realidade pelo ser humano, como grupo ou mesmo pelo indivíduo humano como ser consciente (em questões que se tornam pertinentes ao nos darmos conta de que mesmo a consciência de outros objetos da realidade é tão questionável quanto qualquer instância dessa realidade exterior; alguém poderia se perguntar se qualquer sistema quântico é passível de colapsar sem que esta própria pessoa tenha realizado uma medida). Neste ponto as discussões podem perder o contato com a pertinência científica, mas devemos considerar que a hipótese está contida na elaboração do experimento do gato de Schrödinger.

Os pontos levantados por EPR e Schrödinger não nos dão nova previsão quantitativa e, talvez, devido a isso que essas ideias passaram muito tempo no campo da filosofia. A partir de questionamentos desta natureza e motivado pelo argumento de EPR, Bell propõe uma nova visão para a MO. 


\section{O teorema de Bell}

Anos se passaram após os trabalhos de Bohm0 referentes a variáveis ocultas e a violação da condição de localidade, para que Bell apontasse as limitações na prova de impossibilidade de von Neumann $\underline{71}$ e discutisse a teoria de Bohm como sendo uma característica geral de qualquer teoria de variável oculta consistente com a teoria quântica. No artigo publicado em 1964, Bell derivou uma desigualdade envolvendo observáveis físicos mensuráveis para pares correlacionados de partículas que seria obedecida por qualquer TVOL, mas que seria violada pela teoria quântica. A M0 violava tais desigualdades, como ficou claro anos mais tarde.

Entendendo que, as variáveis ocultas são propostas devido à imposição da condição de localidade sua admissão pode ser considerada como a única forma de evitar os efeitos radicalmente não locais que emergiam como resultados do experimento mental proposto, era o meio para que a teoria oferecesse uma descrição completa da realidade física ${ }^{72}$. Apesar da relevância do argumento apresentado por EPR no contexto das ideias da época, uma teoria local completada por variáveis ocultas não é possível. 0 problema da não-localidade só surge quando se consideram duas partículas correlacionadas, como foi demonstrado por Bell.

No teorema é analisado duas partículas em um estado entrelaçado descritas por um estado singlet com spin total nulo; e toma a premissa einsteniana da separabilidade (independência de sistemas físicos distantes no contínuo espaço-tempo). Assim, fazendo-se uso do princípio da superposição aplicado a sistemas compostos (emaranhados), a M0 produz previsões quantitativas que, se confirmadas (a questão ainda não está fechada), invalidariam ou o realismo ou a localidade, as duas hipóteses utilizadas por Bell para demonstrar sua desigualdade.

Os resultados de uma medição do spin levam à predeterminação das variáveis da partícula não sujeita à medição. Isso implica, como a teoria quântica não representa esta predeterminação, na possibilidade de uma mais completa especificação do sistema. A contribuição essencial de Bell vem a partir deste ponto.

Bell traduz a premissa da separabilidade num critério físico e matemático e denomina de localidade; com base neste critério formula um modelo de uma descrição mais completa, usando variáveis adicionais às usadas pela teoria quântica e busca tirar consequências deste modelo. Em um cálculo simplificado, ele demonstra que um modelo deste tipo são desigualdades (local e realista) que podem ser violadas pelas previsões da MO. Sua conclusão é então que, ao menos no caso considerado, que a TVOL é incompatível com a teoria quântica e, portanto, que a teoria não pode ser "completada" dessa maneira.

Os estados de EPR eram, não-normalizáveis, impossíveis de serem preparados em laboratório. De fato, até o teorema de Bell, a única maneira de explorar a teoria quântica por meio de variáveis ocultas locais, foi o experimento de Chien-Shiung Wu e Irving Shaknov ${ }^{73}$ que envolvia medições relacionadas à polarização de fótons emitidos do aniquilamento de um par pósitron-elétron, compararam as previsões nas quais esse sistema (experimento tipo-EPR para partículas com spin $1 / 2$ ) é descrito por um estado entrelaçado puro com outros dois modelos de misturas locais que envolviam a teoria quântica de polarização. Bell, por sua vez, usou em sua análise do problema da não-localidade/realismo e o sistema quântico emaranhado mais simples possível: duas partículas de spin 1/2 correspondente ao mesmo sistema utilizado por Bohm, segue esquematizado na Figura 1.

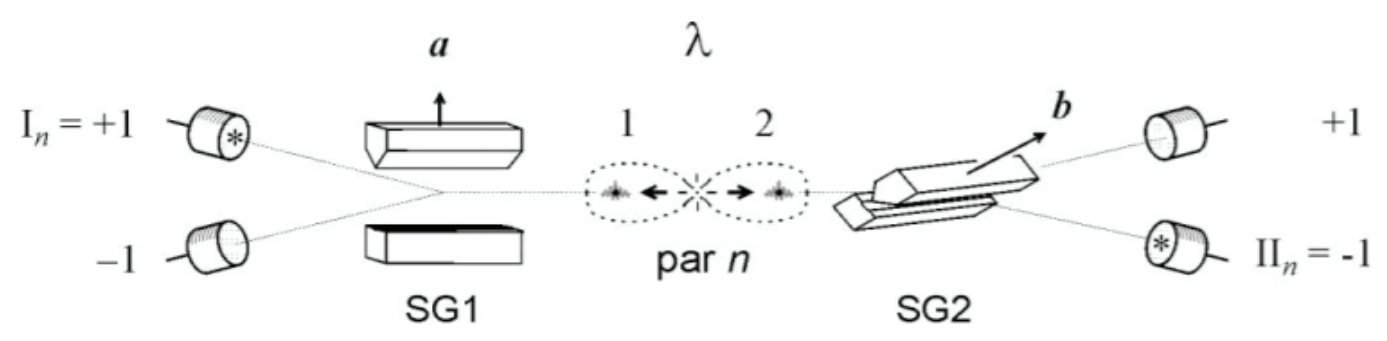

Figura 1: Montagem para a verificação do teorema de Bell. FONTE: PESSOA JR., 0. Introdução histórica à Teoria Quântica, aos seus problemas de fundamento e às suas interpretações. Caderno de física da UEFS 04 (01 e 02): 89-114, 2006. 
As componentes de spin em várias direções são as observáveis representadas por operadores que não comutam, a correlação entre as medições obtidas para os pares de partículas depende dos ângulos escolhidos para a medição dos spins segundo orientação dos aparatos de Stern-Gerlach utilizados. Na Figura 1, vemos os aparatos orientados em direções arbitrárias $a$ e $b$, sendo que o componente de spin tem valor $+\frac{\hbar}{2}$, com simplificação este valor será +1 , e quando for $-\frac{\hbar}{2}$ denota-se -1.0 resultado obtido para a partícula 1, no par $n$, é denotado por $I_{n}$, e o resultado obtido para a partícula 2 é denotado por $I I_{n}$.

Bell reescreve uma equação conhecida como equação de De Broglie-Bohm ${ }^{74}$, para um par de partículas com spins correlacionados, as quais formam um sistema de equações acopladas, exibindo assim um caráter não local. Ele então considerou que, se a função de onda fosse inicialmente fatorável, esta fatorabilidade traria a situação de que o estado de uma das partículas seria independente do estado da outra, o que representa a hipótese de localidade, ou separabilidade. Em geral a função de onda não é fatorável, o que faz com que a teoria de Bohm tenha um caráter não-local, ou seja, a disposição em uma parte do aparelho afeta os resultados obtidos em outra parte distante.

Bell derivou uma de suas desigualdades que seria satisfeita por quaisquer TVOL obtendo um resultado surpreendente: esta desigualdade, em certas circunstâncias, para ângulos específicos, era violada pela M0. Esse resultado mostra uma incompatibilidade entre a MO e quaisquer TVOL, incompatibilidade esta que atualmente é conhecida como Teorema de Bell. A desigualdade de Bell, também chamada de teorema de Bell, é uma família de resultados matemáticos que mostra que as previsões experimentais fornecidas pela teoria quântica não estão de acordo com as fornecidas pela TVOL. A desigualdade apresentada por Bell foi à seguinte:

$$
|P(a, b)-P(a, c)| \leq 1+P(b, c)
$$

Onde $P(a, b), P(a, c)$ e $P(b, c)$ são as funções de correlação entre as duas partículas em várias orientações dos detectores, ou seja, uma média dos produtos das medições realizadas nas duas partículas e 'a', 'b' e 'c' são versores ${ }^{75}$.

Bell propôs que, ao invés de orientar o elétron, os detectores e o pósitron na mesma direção, os detectores girassem independentemente e sugeriu calcular o valor médio dos produtos dos spins, chamando-os de $P(a, b)$. 0 detector $D_{1}$ mede o componente de spin do elétron na direção do versor "a", o detector $D_{2}$ mede o componente de spin do pósitron na direção do versor " $b$ ". Se os detectores $\left(D_{1}\right.$ e $\left.D_{2}\right)$ estiverem paralelos entre si teremos um spin "up" e um spin "down", logo o produto entre eles será sempre -1, consequentemente a média também será -1: $P(a, a)=-1$. Se os detectores forem antiparalelos (ou perpendiculares) o produto será +1 , sendo assim a média dos produtos será: $P(a,-a)=+1$.

Lembremos que essas desigualdades supõem a existência de variáveis ocultas locais. Essas variáveis obedecem à condição de localidade (a medida realizada sobre uma partícula não altera o estado da outra partícula, estando essas separadas espacialmente). 0 que Bell mostrou foi que as previsões da M0 violam essas desigualdades, logo há uma incompatibilidade de resultados entre as previsões teóricas da MO e das TVOL.

Dessa forma, ou a M0 (e, portanto, a natureza) é não-local, ou não-real, ou não-local e não-real. Porém, as violações da desigualdade de Bell não nos permitem dizer qual das duas hipóteses, realismo e/ou não-localidade a M0 não satisfaz, já que ambas são necessárias para se chegar à desigualdade e que podem ser violadas apenas por sistemas emaranhados. Qualquer estado puro emaranhado exibe alguma inconsistência com o realismo local, ou seja, sempre é possível encontrar algum tipo de desigualdade de Bell na qual um estado puro emaranhado viole.

Os experimentos realizados tornaram possíveis os testes de alguns aspectos apenas. 0 realismo e a não-localidade podem se manifestar de outras formas na teoria, não apenas através de teorias de variáveis ocultas. Ressaltamos que, para a verificação experimental das desigualdades de Bell ainda foram necessários outros desenvolvimentos e mesmo hipóteses adicionais às consideradas inicialmente. Assim, os debates sobre o realismo, a não-localidade e a completude da teoria quântica permanecem em aberto. Esses são apenas alguns debates que são pontos de discordância entre as diferentes interpretações da teoria quântica. 
Em particular, experimentos muito importantes foram feitos por Aspect e colaboradores ${ }^{\frac{76}{6}}$, comprovando quase definitivamente que a $\mathrm{MO}$ viola a desigualdade de Bell. Estes resultados, conforme veremos, deixam cada vez menos espaço para a TVOL.

\section{Os experimentos de Aspect e colaboradores}

No debate sobre os fundamentos da teoria quântica marca-se a partir do final da década de 50 como o período que guiou a realização de diversos testes experimentais das desigualdades de Bell. A princípio, o teorema de Bell teve uma repercussão inicial muito discreta, até que um grupo de físicos se debruçou na verificação experimental da desigualdade de Bell.

Bohm e Aharonov ${ }^{77}$, baseados em um experimento realizado em 1950 por Wu e Shaknov, marca as primeiras tentativas de testar experimentalmente os chamados estados "entrelaçados" afirmando que se o componente $x$ é definido, então os componentes y e $z$ são indeterminados. Os autores retomam o argumento de EPR ressaltando a dificuldade que surge a partir do princípio da incerteza da M0, segundo o qual apenas um componente do spin de cada partícula pode ter um valor definido em um dado instante.

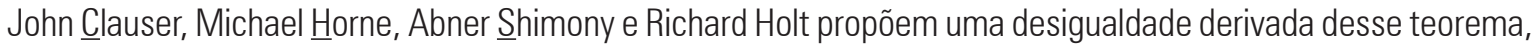
conhecida como $\mathrm{CHSH}^{78}$, que poderia ser testada em laboratórios de óptica quântica. Outras medições foram feitas por Freedman e Clauser a partir de uma transição entre estados de um átomo de cálcio, que resultava na emissão de dois fótons ${ }^{79}$. Em 1976, Edward Fry e Randall Thompson medem a correlação de polarização linear entre os dois fótons. Os resultados foram utilizados para avaliar a versão de Freedman da desigualdade de Bell ${ }^{80}$.

0 ápice deste processo, e o mais rigoroso, foi a série dos três trabalhos do grupo de Alain Aspect que refinou significativamente medidas anteriores usando laser sintonizável para excitar amostras atômicas e obter o par de fótons por decaimento do elétron excitado. Os resultados finais obtêm clara violação da desigualdade e excelente concordância com a previsão da M0, impondo sérias restrições à possibilidade de existência de TVOL que pudessem descrever os sistemas quânticos.

0 conjunto dos experimentos indicava uma violação das desigualdades de Bell, e, portanto, das possíveis teorias realísticas locais, e uma confirmação das previsões da teoria quântica. Após o experimento de Aspect, ampliou-se sensivelmente o número dos que tinham esta mesma avaliação.

0 francês Alain Aspect ${ }^{81}$ liderou duas equipes diferentes que realizaram três experimentos nesta linha, publicando os resultados em 1981 e 1982 em Physical Review Letters. Destes, o terceiro experimento foi inicialmente realizado com polarizadores variando no tempo, seguindo propostas anteriores de Bohm e Aharonov e do próprio Aspect. Este experimento se torna mais importante pelas alterações realizadas em 1982: uso de um aparato que mudasse as configurações experimentais enquanto os fótons estivessem em voo. Bell insistira na importância dos experimentos nos quais suas configurações mudassem enquanto os fótons estivessem em voo, pois estes testariam o caráter não local da natureza, caráter este que supostamente poderia levar a um sinal mais rápido que ao da luz.

A técnica de excitação foi a mesma utilizada no experimento anteriormente analisado ${ }^{82}$, inclusive a cascata e a substância excitada, porém foi necessário diminuir a divergência do feixe, a fim de obter-se um bom funcionamento dos interruptores, já que estes mudavam os fótons rapidamente de um polarizador para outro. Isto implicou na diminuição da quantidade de dados coletados por intervalo de tempo, e na diminuição da taxa de coincidência por segundo. Não foi possível manter a eficiência na coleta de fótons tão boa quanto às anteriores, já que tiveram que reduzir a divergência no feixe para conseguir um bom funcionamento dos interruptores ${ }^{83}$. Este fator é importante, já que este é um experimento estatístico, porém não tirou a confiabilidade do resultado experimental. 
A principal mudança para a análise está na técnica de detecção. Também foram usados quatro fototubos, só que ao invés de dois polarizadores eles utilizaram quatro, um polarizador para cada fototubo, no qual as orientações destes polarizadores eram modificadas após os fótons terem sidos emitidos da cascata de cálcio. Isto se dava através de um dispositivo shutter, que mudava rapidamente a direção dos fótons de um polarizador para o outro, polarizadores estes pertencentes a uma mesma direção "a" ou "b". As mudanças entre os dois polarizadores ocorrem a cada $10 \mathrm{ns,}$ sendo 0 tempo de vida no estado intermediário $5 \mathrm{~ns}$. 0 tempo de mudança entre os polarizadores era menor do que 0 tempo de vôo dos fótons (40 ns), seguindo a proposta de mudança da configuração experimental enquanto os fótons estivessem em voo. No experimento em questão (Figura 2), se viola claramente as desigualdades de Bell.

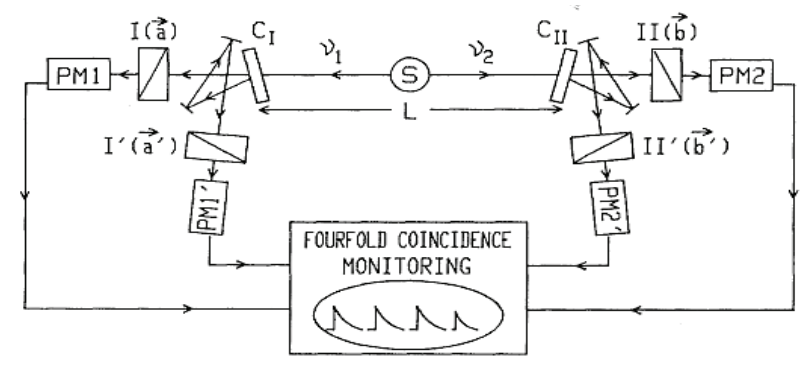

Figura 2: Esquema experimental apresentado por Aspect e colaboradores. FONTE: ASPECT, A.; DALIBARD, J.; ROGER, G. Experimental test of Bell's inequalities using time-varying analyzers. Physical Review Letters, v. 49, n. 25, p. 1804-1807, 1982.

A fonte é representada por " $S$ ", $V_{1}$ e $V_{2}$ são os fótons correlacionados, $C_{\mid}$e $C_{\|}$são os interruptores, " $L$ " é a distância de um interruptor a outro, os polarizadores são representados por I(a) I'(a') II(b) e II(b'), PM1 e PM2 representam os fototubos. 0 valor da quantidade " $\mathrm{S}$ " - fonte que emite simultaneamente um par de partículas no estado spin singleto - encontrada no experimento em questão foi: $S=0,101 \pm 0,020$, mas, o valor máximo para a quantidade "S" é zero. De acordo com as previsões da M0 o valor de "S" é igual a: $S=0,112$. Logo, os resultados desse experimento apontaram para as previsões da M0.

Esses experimentos não foram os últimos testes da MO. Atualmente, Anton Zeilinger realiza outros experimentos, cada vez mais sofisticados, buscando testar de várias formas à confirmação das previsões da M0 para a correlação das polarizações de um par de fótons separados um do outro ${ }^{84}$. Outros estudos surgem com diferentes processos experimentais e demonstraram uma ampla classe de teorias realistas não locais que são incompatíveis com correlações quânticas observáveis $s^{\frac{85}{5}}$.

Desse modo, podemos afirmar que até os dias atuais a M0 ainda é testada. Uma discussão sobre essa continuidade foge dos objetivos do presente texto, sendo uma possibilidade de trabalhos futuros. No entanto, é possível que uma justificativa esteja no fato de que a questão do realismo continua em aberto. Afinal, essa questão não se reduz à existência de variáveis ocultas.

\section{Considerações finais}

Apresentada ao mundo científico em 1927, no $5^{\circ}$ Congresso Solvay, a teoria quântica desencadeou um longo debate, que perdura até os nossos dias, que pode ser caracterizado como o principal debate científico e epistemológico da física do século XX. Na história deste debate, nessa narrativa, foi dado lugar de destaque os trabalhos de Eintein, Podolsky e Rosen, Schrödinger, e Bell, com suas inquietantes desigualdades.

Estes trabalhos tratam do debate a respeito do realismo, da não-localidade e da completude da teoria quântica. Suscitou diversos outros debates sobre questões fundamentais, além de evidenciar o problema da medição. Em conse- 
quência, revelam o processo de construção do conhecimento científico que transcorre a epistemologia, a matemática e a experimentação.

Conforme discutido ao longo do trabalho, tomamos por base alguns trabalhos do período de 1935 até 1982 , devido ao desenvolvimento do argumento de EPR, sendo o artigo de 1935 um marco importante no esclarecimento das questões envolvidas nesse debate. A análise histórica dos textos escolhidos nos informa que os vários argumentos para que se superasse as contradições observadas na $\mathrm{MO}$ e que a atividade experimental foi crucial para a aceitação definitiva da teoria quântica.

A partir da década de 1990, tornou-se evidente que estados quânticos emaranhados (emaranhamento quântico), além de serem interessantes para discussões sobre as bases conceituais da física, podem ser vistos como um recurso da natureza. Os estados emaranhados podem ser usados para se realizar eficientemente tarefas impossíveis de serem executadas por meio de recursos clássicos. Atualmente, o emaranhamento quântico passa a ser visto como um recurso em potencial para desenvolvimento tecnológico, como nas áreas de Computação Quântica e Informação Quântica, por exemplo.

Por fim, essa narrativa histórica torna-se interessante para estudantes/professores de física e entusiastas da teoria quântica, nos seus vários níveis de formação, tendo em vista que, apesar da importância das discussões sobre determinismo, realismo e não-localidade, quase todos os livros de texto voltados a aprendizagem da $\mathrm{MO}$, com raras exceções, ignoram relevantes aspectos - em consequência, supre de algum modo lacunas quanto falta de materiais didáticos, além de contribuir para compreensão conceitual significativa da M0. Notemos também que optamos por um recorte histórico frente à $\mathrm{M0}$, destacando figuras importantes, bem como seus trabalhos. Outros recortes podem ser feitos sem prejuízo à compreensão dos fenômenos quânticos.

\section{Notas e referências bibliográficas}

Rafaelle da Silva Souza é professora de Física do IFBA campus Seabra e doutoranda do Programa de Pós-Graduação em Ensino, Filosofia e História da Ciência - UFBA/UEFS. Agradece o apoio financeiro da CAPES. E-mail: rafaellesouza2@yahoo.com.br.

Indianara Silva é professora do departamento de Física da UEFS e vinculada ao Programa de Pós-Graduação em Ensino, Filosofia e História da Ciência - UFBA/ UEFS. E-mail: indianara.slima@gmail.com.

Elder Sales Teixeira é professor do departamento de Física da UEFS e vinculado ao Programa de Pós-Graduação em Ensino, Filosofia e História da Ciência - UFBA/ UEFS. E-mail: eldersate@gmail.com.

1 BELL, J. S. On the Einstein-Podolsky-Rosen Paradox. Physics, v. 1, p. 195-200, 1964

2 FREIRE JR., O. A story without an ending: the quantum physics controversy 1950-1970. Science \& Education, v. 12, n. 5, p. 573-586, 2003; Quantum dissidents: Research on the foundations of quantum theory circa 1970. Studies in History and Philosophy of Science Part B, v. 40, n. 4, p. $\overline{280-289}$ 2009; ___ Philosophy enters the optics laboratory: Bell's theorem and its first experimental tests (1965-1982). Studies in History and Philosophy of Science Part B, v. 37, n. 4, p. 577-616 2006.

3 Sobre a interpretação de histórias consistentes, ver: GRIFFITHS, R.B. Consistent Histories and the Interpretation of Quantum Mechanics. Journal of Statistical Physics, v. 36, p. 219-72, 1984; OMNÈS, R. Consistent Interpretations of Quantum Mechanics. Reviews of Modern Physics, v. 64, p. 339-82, 1992.

4 PESSOA JR., 0. Mapa das Interpretações da Teoria Quântica. In: MARTINS, R. A.; BOIDO, G.; RODRíGUEZ, V. (orgs.). Física: Estudos Filosóficos e Históricos. Campinas: AFHIC, 2006a, pp. 119-52.

5 MEHRA, J. e RECHENBERG, H. The Historical Development of Quantum Theory. New York: Springer, 1982, p. 426.

6 Destacamos: JAMMER, M. The Conceptual Development of Quantum Mechanics. New York: McGraw-Hill, 1966; KRAGH, H. Quantum generations: a history of physics in the twentieth century. Princeton: Princeton University Press, 1999.

$7 \quad$ TIPLER, P. A. e LLEWELLYN, R. A. Física Moderna, $6^{a}$ ed. Rio de Janeiro: LTC, 2001.

8 COHEN-TANNOUDJI, C.; DIU, B.; LALOË, F. Quantum Mechanics. vol. I. New York: John Wiley \& Sons, 2006.

9 CARUSO, F.; OGURI, V. Física Moderna. Origens Clássicas \& Fundamentos Quânticos. 2ª ed. São Paulo: Elsevier, 2009.

10 GRIFFITHS, D. J. Mecânica Quântica. 2ed. São Paulo: Pearson, 2011.

11 Segue lista de referências que discorrem sobre os problemas enfrentados no ensino de Mecânica Quântica devido à má qualidade do material didático: CID, M., R. e DA SILVA, A. Estudiando cómo los modelos atómicos son introducidos en los libros de texto de Secundaria. Revista Eureka sobre Enseñanza y Divulgación de las Ciencias, v. 9, p. 329-337, 2012. FANARO, M. La enseñanza de la mecánica cuántica en la Escuela Media (tesis de doctorado). Universidad de Burgos, Burgos, Espanha, 2009. DOMENECH, J., SAVALL, F. e MARTínEZ, J. La introducción del concepto de fotón en bachillerato. Revista Brasileira de Ensino de Física, v. 35, p. 1-14, 2013. SINARCAS, V. e SOLBES, J. Dificultades en el aprendizaje y la enseñanza de la física cuántica en el 
bachillerato. Enseñanza de las Ciencias, v. 31, p. 9-25, 2013; VIZCAINO, D. Significados de matematización en la enseñanza de la física presentes en libros didácticos de física universitaria. Tecné, Episteme y Didaxis, número extraordinario, 908-914, 2016.

12 HENDRY, J. The creation of quantum mechanics and the Bohr-Pauli dialogue. Dordrecht: Reidel, 1984

13 BELLER, M. Quantum dialogue. The making of a revolution. Chicago: University of Chicago, 1999.

14 DARRIGOL, O. A simplified genesis of quantum mechanics. Studies in History and Philosophy of Modern Physics, v. 40, p. 151-166, 2009.

15 MILLAR, R.; OSBORNE, J. Beyond 2000: science for the future. London: King's College, 1998.

16 NORRIS, S.P.; GUILBERT, S.M.; SMITH, M.L.; HAKIMELAHI, S.; PHILLIPS, L.M. A Theoretical Framework for Narrative Explanation in Science. Science Education, v. 89, n. 4, p. 535-563, 2005.

17 METZ, D.; KLASSEN, S.; MCMILLAN, B.; CLOUGH, M.; OLSON, J. Building a Foundation for the Use of Historical Narratives. Science \& Education 16: pp. 313-334, 2007.

18 KLASSEN, S. The application of Historical Narrative in Science Learning: The Atlantic Cable Story. Science \& Education, v. 16, pp. 335-352, 2006.

19 VON NEUMANN, J. Mathematische Grundlagen der Quantenmechanik. Berlin: Springer, 1932. Tradução em inglês: Mathematical Foundations of Quantum Mechanics. Princeton: Princeton University Press, 1955. (Referências indicam a tradução).

20 PINTO NETO, N. Teorias e interpretações da Mecânica Quântica. São Paulo: Editora Livraria da Física; Rio de Janeiro: CBPF, 2010.

21 MARTINS, R. A. Como não escrever sobre história da física: um manifesto historiográfico. Revista Brasileira de Ensino de Física, v. 23, n. 1, p. 113-129, 2001.

22 FORATO, T. C. M. A Natureza da Ciência como Saber Escolar: um estudo de caso a partir da história da luz. Tese (Doutorado em Educação) - USP, São Paulo, 2009.

$23 \mathrm{KRAGH}$, H. An introduction to the historiography of science. Cambridge: Cambridge U.P., 1987.

24 NORRIS et al., op. cit., 2005.

25 KLASSEN, op. cit., 2006

26 METZ et al., op. cit., 2007.

27 MARTINS, A. F. P. Natureza da Ciência no ensino de ciências: uma proposta baseada em "temas" e "questões". Centro de Educação - UFRN. Caderno Brasileiro de Ensino de Física, v. 32, n. 3, p. 703-737, 2015.

28 ALLCHIN, D.; ANDERSEN, H. M.; NIELSEN, K. The episodic historical narrative as a structure to guide inquiry in science and nature of science education. In: INTERNATIONAL CONFERENCE ON HISTORY OF SCIENCE \& SCIENCE EDUCATION, 10., 2014, Minneapolis, MN.

29 EINSTEIN, A.; PODOLSKY, B.; ROSEN, N. Can quantum-mechanical description of physical reality be considered complete? Physical Review, v. 47, p. 777780, 1935.

30 SCHRÖDINGER, E. Die gegenwärtige Situation in der Quantenmechanik. Naturwissenschaften, v. 23, p. 807-812; 823-828; 844-849, 1935.

31 BELL, op. cit., 1964.

32 ASPECT, A., DALIBARD, J. e ROGER, G. Experimental test of Bell's inequalities using time-varying analyzers. Physical Review Letters, v. 49, n. 25, p. 1804-1807, 1982.

33 PESSOA JR., 0. Introdução histórica à Teoria Quântica, aos seus problemas de fundamento e às suas interpretações. Caderno de física da UEFS, v. 4, n. 1-2, p. 89-114, 2006.

34 Relatos com maiores detalhes sobre esses aspectos são apresentados por: JAMMER, M. The Philosophy of Quantum Mechanics. New York: Wiley, 1974.

35 PESSOA JR., 0. 0 problema da medição em Mecânica Quântica: um exame atualizado. Cadernos de História e Filosofia da Ciência (série 3), v. 2, n. 2, p. 177-217, 1992.

36 FREIRE JR., 0.; CARVALHO NETO, R. A. 0 universo dos quanta: uma breve história da Física Moderna. São Paulo: FTD, 1997.

37 PESSOA JR., op. cit., 1992.

38 PESSOA JR., op. cit., 2006.

39 WIGNER, E.P. The Problem of Measurement. American Journal of Physics, v. 31, p. 6-15, 1963.

40 BELL, J. S. On the Problem of Hidden Variables in Quantum Mechanics. Reviews of Modern Physics, v. 38, p. 447-452, 1966.

41 Tradução para o português pode ser encontrada em: Cadernos de História e Filosofia da Ciência v. 2, p. 90-96, 1981.

42 BOHR, N. Can quantum-mechanical description of physical reality be considered complete? Physical Review, v. 48, n. 8, p. 696-702, 1935.

43 SCHRÖDINGER, op. cit., 1935.

44 BOHM, D. A suggested interpretation of the quantum theory in terms of "hidden" variables I. Physical Review, v. 85, n. 2, p. 166-179, 1952a; A suggested interpretation of the quantum theory in terms of "hidden" variables II. Physical Review, v. 85, n. 2, p. 180-193, 1952b.

45 Um relato claro e compreensivo da teoria de Bohm e sua recepção é dado por CUSHING, James T. Quantum Mechanics: Historical Contingency and the Copenhagen Hegemony. Chicago: University of Chicago Press, 1994.

46 BELL, op. cit., 1964.

47 CLAUSER, J., HORNE, M., SHIMONY, A., e HOLT, R. Proposed experiment to test local hidden-variable theories. Physical Review Letters, v. 23, n. 15, p. 880-884, 1969.

48 FREEDMAN, S. J. e CLAUSER, J. F. Experimental Test of Local Hidden-Variable Theories. Physical Review Letters, v. 28, p. 938-941, 1972.

49 ASPECT, A., DALIBARD, J. e ROGER, G. Experimental test of Bell's inequalities using time-varying analyzers. Physical Review Letters, v. 49, n. 25, p. 1804-1807, 1982.

50 BISPO, W. F. O.; DAVID, D. F. G. Sobre a cultura material dos primeiros testes experimentais do teorema de Bell: uma análise das técnicas e dos instrumentos (1972-1976). In: FREIRE JR, O., PESSOA JR, 0., BROMBERG, JL., orgs. Teoria Quântica: estudos históricos e implicações culturais. Campina Grande: EDUEPB; São Paulo: Livraria da Física, 2011. 
ASPECT, A. One century of quantum revolutions. In: SCARANI, V. Quantum physics, a first encounter: interference, entanglement, and reality. Oxford: Oxford University Press, 2006, p. ix-xiv.

53 Uma discussão mais extensa e precisa das sobre as posições filosóficas de Einstein, ver: PATY, M. Einstein. São Paulo: Estação Liberdade, 2008.

54 EINSTEIN, PODOLSKY, ROSEN, op. cit., 1935, p. 777.

55 Idem, p. 779

$56 \quad$ Idem, p. 777.

$57 \quad$ Idem, p. 778.

58 Não se tem a pretensão de explicar minuciosamente este processo e sim exemplificá-lo de forma simples. Para um maior entendimento ver: PESSOA JR., 0. Conceitos de Física Quântica, v.2. São Paulo: Editora Livraria da Física, 2003.

$59 \Sigma \mathrm{IC}_{i} \mathrm{I}^{2}=1$ significa dizer que a função é normalizada, i.e., a probabilidade total é igual a um.

60 "Após a medição, o sistema passa a se encontrar em um novo estado, estado este que depende do resultado obtido. Assim, pode-se dizer que no decorrer da medição o sistema evoluiu de maneira indeterminista. Esta transição tem sido chamada de 'colapso do pacote de onda' ou 'redução de estado', sendo descrita pelo postulado da projeção de von Neumann" (PESSOA Jr., op. cit., 1992, p. 178).

61 EINSTEIN, PODOLSKY, ROSEN, op. cit., 1935, p. 779.

62 Idem, p. 780.

63 Segundo Howard, a incompletude de Einstein apresentava argumentos diferentes dos de Podolsky, conforme pode ser verificado em carta escrita a Schrödinger no mesmo ano em que 0 artigo original foi escrito. Esses aspectos influenciam diretamente na compreensão dos critérios de completude e na noção de elemento de realidade. Para outras referências que discutem a versão original de Einstein para o EPR, para saber mais ver: HOWARD, D. Einstein on locality and separability. Studies in History and Philosophy of Science, v. 16, 171-201, 1985.

64 CHIBENI, S. Aspectos da descrição física da realidade. Campinas: Centro de Lógica, Epistemologia e História da Ciência, 1997.

65 EINSTEIN, PODOLSKY, ROSEN, op. cit., 1935, p. 778.

66 Tradução para o português por Cláudio Weber Abramo: Cadernos de História e Filosofia da Ciência, v. 2, p. 97-106, 1981.

67 Outro viés de discussão, com igual relevância, é considerar o debate Einstein-Bohr, ver: BROWN, H. R. 0 debate Einstein-Bohr sobre a mecânica quântica. Cadernos de História e Filosofia da Ciência, v. 2, p. 51-89, 1981.

68 SCHRÖDINGER, op. cit., 1935.

69 Idem, p. 157.

70 Em 1951 David Bohm havia simplificado em seu livro os argumentos de EPR usando uma descrição de sistema composto mais simples do que o usado por EPR. BOHM, David. Quantum Theory. New York: Prentice-Hall, 1951.

71 Bell não foi o primeiro a apontar a falha na prova de impossibilidade de von Neumann. Grete Hermann, filósofa e matemática alemã, em 1935 (30 anos antes de Bell) já havia apontado falhas semelhantes a Bell no trabalho de von Neumann. Porém, seus apontamentos foram ignorados pela comunidade de físicos. GREENSTEIN, G.; ZAJONC, A. The quantum challenge: modern research on the foundations of quantum mechanics. Sudbury: Jones and Bartlett, 2006.

73 WU, C. S. e SHAKNOV, I. The angular correlation of scattered annihilation radiation. Physical Review, v. 77, p. 136, 1950.

74 Sobre esta equação ver PESSOA, op. cit., 2006, p. 235, nota 17.

75 RIBEIRO FILHO, A. Notas de Aula, curso ministrado para alunos de Pós-Graduação da Universidade Federal da Bahia no segundo semestre de 2008.

76 ASPECT, A., GRANGIER, P. e ROGER, G. Experimental test of realistic local theories via Bell's Theorem. Physical Review Letters, v. 47, p. 460-463, 1981; ASPECT, A., DALIBARD, J., e ROGER, G. Experimental realization of Einstein-Podolsky-Rosen-Bohm gedankenexperiment: A new violation of Bell's inequalities. Physical Review Letters, v. 49, p. 91-94, 1982a; ASPECT, A., DALIBARD, J. e ROGER, G. Experimental test of Bell's inequalities using timevarying analyzers. Physical Review Letters, v. 49, p. 1804-1807, 1982b.

77 BOHM, D. e AHARONOV, Y. Discussion of Experimental Proof for the Paradox of Einstein, Rosen, and Podolsky. Physical Review, v. 108, p. $1070,1957$.

78 CLAUSER, J.F. Early History of Bell's Theorem and Experiment. In: BLACK, T.D. et al. (orgs.). Foundations of Quantum Mechanics. Singapore: World Scientific, 1991, pp. 168-74.

79 Sobre as contribuições de John Clauser para o primeiro teste experimental do teorema de Bell, ver: BISPO, W. F. O.; DAVID, D. F. G.; FREIRE Jr. O. As contribuições de John Clauser para o primeiro teste experimental do teorema de Bell: uma análise das técnicas e da cultura material. Revista Brasileira de Ensino de Física, v. 35, n. 3, p. 3603, 2013.

80 FRY, E. S.; THOMPSON, R. C. Experimental Test of Local Hidden-Variable Theories. Phys. Rev. Lett., v. 37, p. 465, 1976.

81 Para um perfil mais detalhado de Aspect ver FREIRE, op. cit., 2006, pp. 606-610.

82 ASPECT, A., DALIBARD, J., ROGER, G., op. cit., 1982a.

83 ASPECT, A., GRANGIER, P., ROGER, G., op. cit, 1981; ASPECT, A., DALIBARD, J., ROGER, G., op. cit, 1982a.

84 FREIRE JR, 0. The Quantum Dissidents: Rebuilding the Foundations of Quantum Mechanics (1950-1990). New York: Springer, 2015.

85 Para saber mais, ver: GRÖBLACHER, S., PATEREK, T., KALTENBAEK, R., BRUKNER, C., ZUKOWSKI, M., ASPELMEYER, M., e ZEILINGER, A. An experimental test of non-local realism. Nature, v. 446, p. 871-875, 2007; WEIHS, G., JENNEWEIN, T., SIMON, C., WEINFURTER, H., ZEILINGER, A. Violation of Bell's inequality under strict Einstein locality conditions. Physical Review Letters, v. 81, p. 5039-5043, 1998; ROWE, M. A., KIELPINSKI, D., MEYER, V., SACKETT, C. A., ITANO, W. M., MONROE, C., e WINELAND, D. J. Experimental violation of a Bell's inequality with efficient detection. Nature, v. 409, p. 791-794, 2001.

[Artigo recebido em Maio de 2020. Aceito para publicação em Setembro de 2020] 